\title{
Agents for the treatment of fatty liver disease: focus on essential phospholipids
}

\author{
Asad Izziddin Dajani ${ }^{1}$ (D) Adnan Abuhammour ${ }^{2}$ (D)
}

Accepted: 28 April 2021 / Published online: 10 May 2021

(c) The Author(s) 2021

\begin{abstract}
Nonalcoholic fatty liver disease (NAFLD) is the most common chronic liver disease in developed countries, with a prevalence that is increasing. In addition to being a disease of the liver, NAFLD is associated with an increased risk of type 2 diabetes mellitus, chronic kidney disease, and cardiovascular disease, with the latter being the most common cause of death in patients with NAFLD. A large number of agents have been trialed in patients with NAFLD or nonalcoholic steatohepatitis (NASH), with or without comorbidities. However, no pharmacologic agents are yet specifically licensed for the treatment of NAFLD. Current treatment guidelines focus on lifestyle modification (diet and exercise, aiming for weight loss) and pharmacologic treatment of comorbidities. Some guidelines suggest the use of pioglitazone (off-label for nondiabetic patients) or vitamin E in select patients with NASH; however, they also note that there are safety concerns with these agents. Essential phospholipids (EPLs) have been safely used for many years as hepatoprotection in patients with liver disease and are recommended as supportive treatment by some NAFLD treatment guidelines. In this narrative review, we discuss the evidence surrounding the use of various agents as treatments in patients with NAFLD or NASH, with a focus on EPL.
\end{abstract}

\section{Key Points}

Although nonalcoholic fatty liver disease is a huge burden on healthcare systems, no pharmacologic agents are yet specifically licensed for this condition.

Some guidelines suggest the use of agents that limit hepatic damage as a potential treatment option.

Many small-scale studies show that essential phospholipids are a promising supportive treatment for these patients; further large well-designed trials are required to confirm these findings.
Asad Izziddin Dajani

aidajani@emirates.net.ae

1 ADSC (Asad Dajani Specialized Center), King Faisal Road next to Medcare Hospital, Al Marzouqi Towers Block C \#605/6, PO Box 6328, Sharjah, UAE

2 Medrise Medical Center, Dubai Health Care City, Dubai, UAE

\section{Introduction}

Nonalcoholic fatty liver disease (NAFLD), the most common chronic liver disease in developed countries, is characterized by the accumulation of liver fat in the absence of competing etiologies [1]. The worldwide prevalence of NAFLD has been estimated at approximately 25\% [2, 3]; however, it is markedly higher in patients who are obese or have type 2 diabetes mellitus or dyslipidemia [3-5]. As the prevalence of obesity and accompanying complications continues to rise, so does the prevalence of NAFLD $[3,6]$. It has been predicted that, in the USA, the prevalence of NAFLD will increase by $21 \%$ from 2015 to 2030 [6].

Isolated steatosis (or nonalcoholic fatty liver [NAFL]) is characterized by fat accumulation in at least $5 \%$ of hepatocytes and may be associated with mild chronic inflammation $[1,5]$. Up to $25 \%$ of patients with NAFLD will progress to nonalcoholic steatohepatitis (NASH), which can be defined as hepatic steatosis with histologic lobular inflammation and ballooning degeneration of hepatocytes [3, 5]. NAFLD can also lead to liver cirrhosis, fibrosis, hepatocellular carcinoma, or end-stage liver disease [5]; some patients with NAFLD may develop cancer without going through fibrosis or cirrhosis, so intervention is needed to treat early-stage NAFLD. Therefore, the goals of therapy in NAFLD are to 
prevent or slow the development of liver damage and to prevent the development of comorbidities, including cancer and cardiovascular disease [7].

Liver biopsy is the gold standard in the diagnosis of NASH. Additionally, the presence of metabolic syndrome is a strong predictor for the presence of steatohepatitis in patients with NAFLD, and physicians should exercise a high level of awareness of the chance of NAFLD and NASH in patients with type 2 diabetes mellitus [2]. According to the NASH Clinical Research Network, diagnostic categories for NAFLD are as follows:

- Not NAFLD ( $<5 \%$ steatosis, by definition)

- NAFL, not NASH ( $\geq 5 \%$ steatosis, with or without lobular and portal inflammation)

- Borderline steatohepatitis, zone 3 or borderline steatohepatitis, zone 1 (most, but not all criteria for steatohepatitis present, with accentuation of steatosis or injury in zone 3 or zone 1, respectively)

- Definite steatohepatitis (all criteria present, including steatosis, hepatocellular ballooning, and lobular inflammation)

The amount of fibrosis can vary across these categories from zero to any amount (up to cirrhosis). Stage 1 is zone 3 (perivenular), perisinusoidal, or periportal fibrosis; stage 2 is both zone 3 and periportal fibrosis; stage 3 is bridging fibrosis with nodularity; and stage 4 is cirrhosis [2].

NALFD is not simply a disease of the liver; it is also an independent risk factor for type 2 diabetes mellitus and cardiovascular disease $[3,5]$. NAFLD increases the risk of cardiovascular mortality [3, 5, 8], and cardiovascular disease is the most common cause of death in patients with NAFLD, with liver-related complications the second most common cause [9]. NALFD is also associated with an increased risk of chronic kidney disease, even after taking potential confounders into account $[10,11]$.

Although NAFLD presents a significant healthcare burden, no direct pharmacologic treatments are yet specifically licensed for this condition [3]. Current management of NAFLD involves lifestyle intervention and pharmacologic treatment of comorbidities [5]. Lifestyle intervention generally focuses on weight loss, which can result in reduced liver fat [5]. However, implementing and maintaining diet and exercise programs to achieve weight loss is often challenging [5]. Additionally, although the majority of patients with NAFLD are overweight, a proportion of them do have a normal body mass index (BMI), with their disease referred to as lean NAFLD [3]. Some recently published treatment guidelines from China and Latvia suggested the possibility of using pharmacological and nutraceutical substances to limit hepatic damage (sometimes referred as "hepatoprotective" substances) in patients with NAFLD [12-14]. One such agent is the highly purified extract of polyenylphosphatidylcholine (PPC) from soybeans, which has been used for many years in patients with liver disease $[13,15]$. This essential phospholipid (EPL) formulation is now a recommended option in NAFLD treatment guidelines from Russia [16], China [12], Latvia [14], and Poland [17], and, in 2017, EPL accounted for $>45 \%$ of the "hepatoprotective" agents sold in Russia [13]. The aim of this narrative review is to discuss the use of these agents as supportive treatment in patients with NAFLD or NASH, with a focus on the data surrounding the use of EPL.

\section{Search methodology}

A search of PubMed and Embase databases was undertaken (from inception through to August 2018 initially and updated in December 2020) to identify studies in which EPL was used to treat NAFLD, NASH, or nonspecified fatty liver disease. Relevant medical subject heading $(\mathrm{MeSH})$ terms were used for the disease names and for EPL as well as possible brand names and generic names. We also conducted a search of clinical databases for gray literature from China and Russia, where EPL is widely used for these indications. The results were manually reviewed to identify prospective clinical studies in which EPL was administered to patients with NAFLD/NASH or nonspecified fatty liver disease.

A similar search of PubMed was also undertaken to identify research relevant to other "hepatoprotective" agents in the treatment of NAFLD/NASH for a brief overview of other agents that may potentially limit hepatic damage.

\section{Overview of essential phospholipid (EPL) and literature search results}

As described earlier, EPL is the highly purified extract of PPC from soybeans. The probable mechanism through which EPL limits liver damage has been described in animals [18]. Phospholipids are essential components of mammalian cells and lipoproteins [19], which are necessary for the formation, regeneration, and activity of membranes, including those of hepatocytes. In in vitro and animal studies, EPL has been shown to restore membrane structure and fluidity, inhibit or correct fibrotic processes, influence apoptosis, and modulate lipid metabolism and has also been shown to have antiinflammatory and antioxidant properties [15].

Russian NAFLD guidelines recommend EPL $1800 \mathrm{mg} /$ day $(2 \times 300 \mathrm{mg}$ capsules, three times a day) for 12-24 weeks as supportive treatment for patients with NAFLD [16]. Latvian NAFLD guidelines also state that the antioxidant and antifibrotic effects of EPL makes their use justified in patients with NAFLD or NASH [14]. Chinese guidelines 
recommend that patients with biopsy-proven NASH, or those suspected to have NASH or advanced fibrosis, should be considered for therapy to limit liver damage, and EPL is mentioned as one option [12]. However, the guidelines state that there is insufficient evidence to recommend one option over another and suggest that patients use one of the recommended agents for a year and monitor for effectiveness (reduction of serum aminotransferase levels) [12]. The Polish guidelines state that oxidative stress is one of the most important links to the pathogenesis of NASH and includes EPL in its list of antioxidant agents but states that most of these agents do not have enough evidence to recommend their use [17]. In a survey study conducted in Polish doctors ( $N=1322$, mostly gastroenterologists and internal medicine specialists), EPL was the most recommended therapy (49\%) for patients with NAFLD, followed by ursodeoxycholic acid (UDCA, 48\%) [20].

The literature search identified 49 studies investigating oral EPL in patients with NAFLD/NASH or nonspecified fatty liver disease without other liver pathologies and with or without comorbidities, including diabetes mellitus and obesity. Of these, we excluded 29 trials in which nonstandard "hepatoprotective" agents were used (for example, Chinese herbal products) or did not focus sufficiently on NAFLD. The 20 remaining trials are outlined in Tables 1,2 and 3. Of these, 12 studies were conducted in China [21-32], four were conducted in Russia [33-36], and one each in the UAE [37], India [38], Poland [39], and Italy [40]. In total, 16 of the studies were randomized [21-32, 34, 35, 38, 39]; of these, two were double-blind trials [38, 39], one was a single-blind controlled trial [25], and the others were open label or did not describe blinding [21-24, 26-32, 34, 35]. The four non-randomized studies were open-label [24, 33, $36,40]$. The duration of the trials ranged from 2 weeks to 24 months, and the EPL dosage, where stated, was most commonly $456 \mathrm{mg}$ three times a day ( $1368 \mathrm{mg} /$ day) [22-26, 28-30, 32, 34], followed by $1800 \mathrm{mg} /$ day [33, 35, 37, 39, 40] or $1800 \mathrm{mg}$ three times a day [36]. Data for one of the prospective observational studies were available from the primary publication of study results [36] and subsequent secondary publications $[41,42]$.

\section{Efficacy}

Common efficacy endpoints in the trials included the following: resolution/improvement of symptoms, hepatic ultrasound, liver function markers (including alanine aminotransferase [ALT], aspartate aminotransferase [AST], and gamma glutamyl transferase [GGT]), serum lipid markers, liver/spleen computed tomography (CT) ratio, and overall response rate (based on one or more of the aforementioned endpoints) [22, 25, 27, 29]. Overall response rate was generally defined as the proportion of patients in which treatment was highly/markedly effective plus the proportion in which it was effective plus, in some trials, as the proportion of cured patients. The definitions of these criteria differed between the papers; however, most were similar to the following:

- Cured: symptoms resolved completely, normal liver function and blood lipid markers, B-mode ultrasonography showing normal liver size/morphology

- Highly/markedly effective: clinical signs significantly improved or disappeared, liver function and blood lipid markers restored or close to normal, B-mode ultrasonography showing normal liver size/morphology

- Effective: clinical signs somewhat improved, liver function and blood lipid markers restored or significantly lowered, liver size/morphology improved

- Ineffective: above criteria worsened or did not improve

In the majority of these studies, EPL treatment (as monotherapy or in combination with other agents) had a positive effect on the outcomes assessed in patients with NAFLD (Table 1), NASH (Table 2), or other/nonspecific fatty liver disease (Table 3).

\section{Nonalcoholic Fatty Liver Disease (NAFLD)}

Of the 17 studies in patients with NAFLD, 11 were in patients with NAFLD alone and six were conducted in patients who had comorbidities (Table 1). Overall, EPL improved NAFLD symptoms, liver function parameters, and liver ultrasonographic features of NAFLD from baseline in both types of study population.

Two noncomparative $\geq 24$-week trials $[36,37]$ provided evidence supporting the use of EPL for the treatment of NAFLD both in patients with NAFLD alone (group A in the trial by Dajani et al. [37]) and in those with NAFLD and comorbidities: type 2 diabetes mellitus or mixed hyperlipidemia (groups B and C, respectively) in the Dajani et al. [37] trial and type 2 diabetes mellitus, obesity, hypercholesterolemia, or hypertension in the MANPOWER trial [36]. Indeed, most patients in the latter trial had two or more comorbidities (79.6\% of 2843 patients) [36]. After 6 months in the Dajani et al. [37] trial, transaminase levels were reduced in $80.5 \%$ of patients in group A (mean reduction in ALT and AST, 54.6 and 48.7 IU, respectively), 84.1\% of patients in group B (ALT - 44.9 IU; AST - 40.5 IU), and in $87.5 \%$ of patients in group C (ALT - 52.9 IU; AST - 49.2 IU). After the EPL dose reduction, the transaminase levels rose slightly but decreased again to normal or just above the upper limit of normal after 3 months $(P<0.01)$. Liver ultrasonography after 6 months showed an improvement in $29.2 \%$ of patients in group A, $23.4 \%$ in group B, and $20.2 \%$ in group $C$; of these, $5.3 \%$ of patients in group $\mathrm{A}, 3.7 \%$ in group $\mathrm{B}$, and $4.8 \%$ in group $\mathrm{C}$ had normal liver 


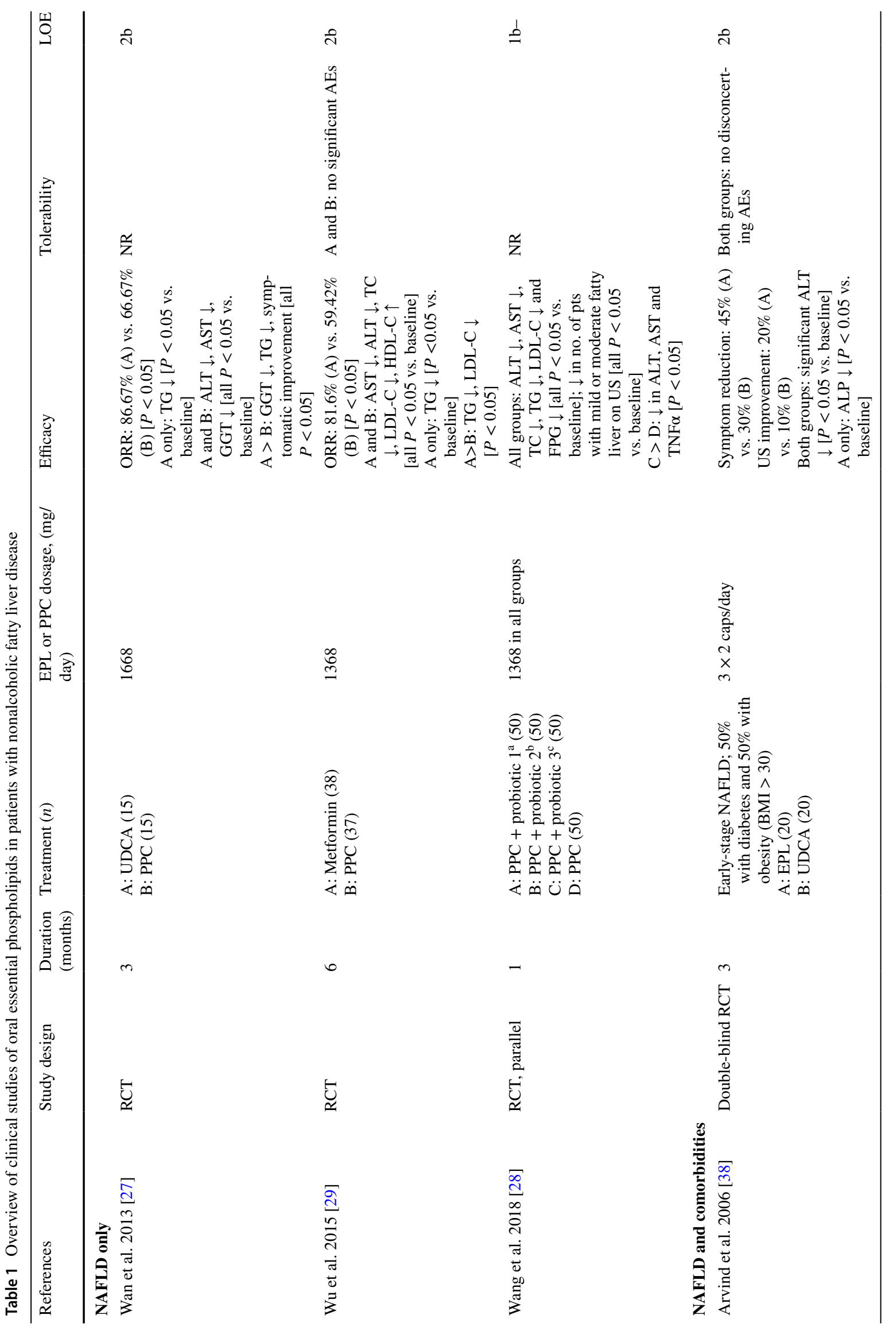




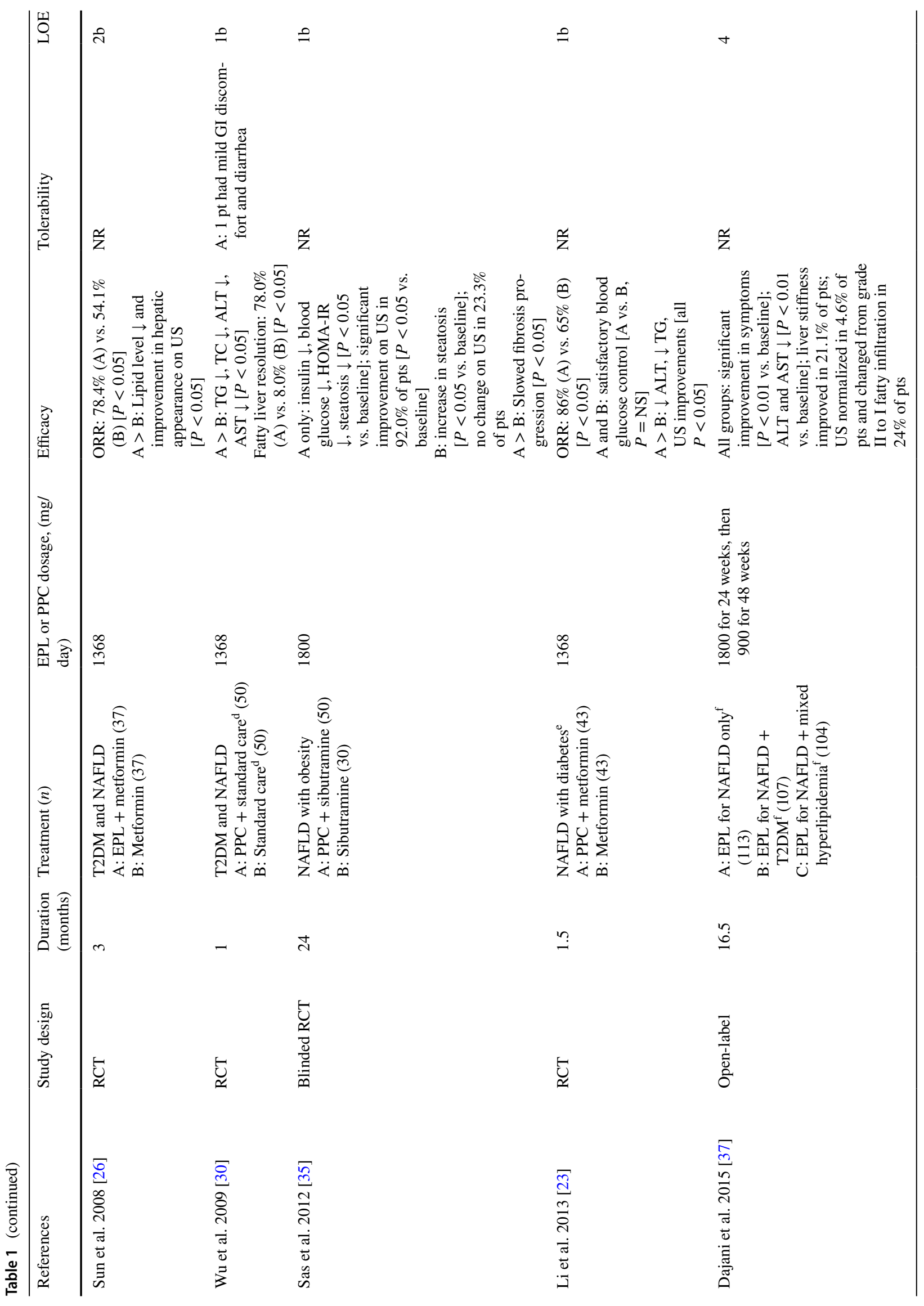




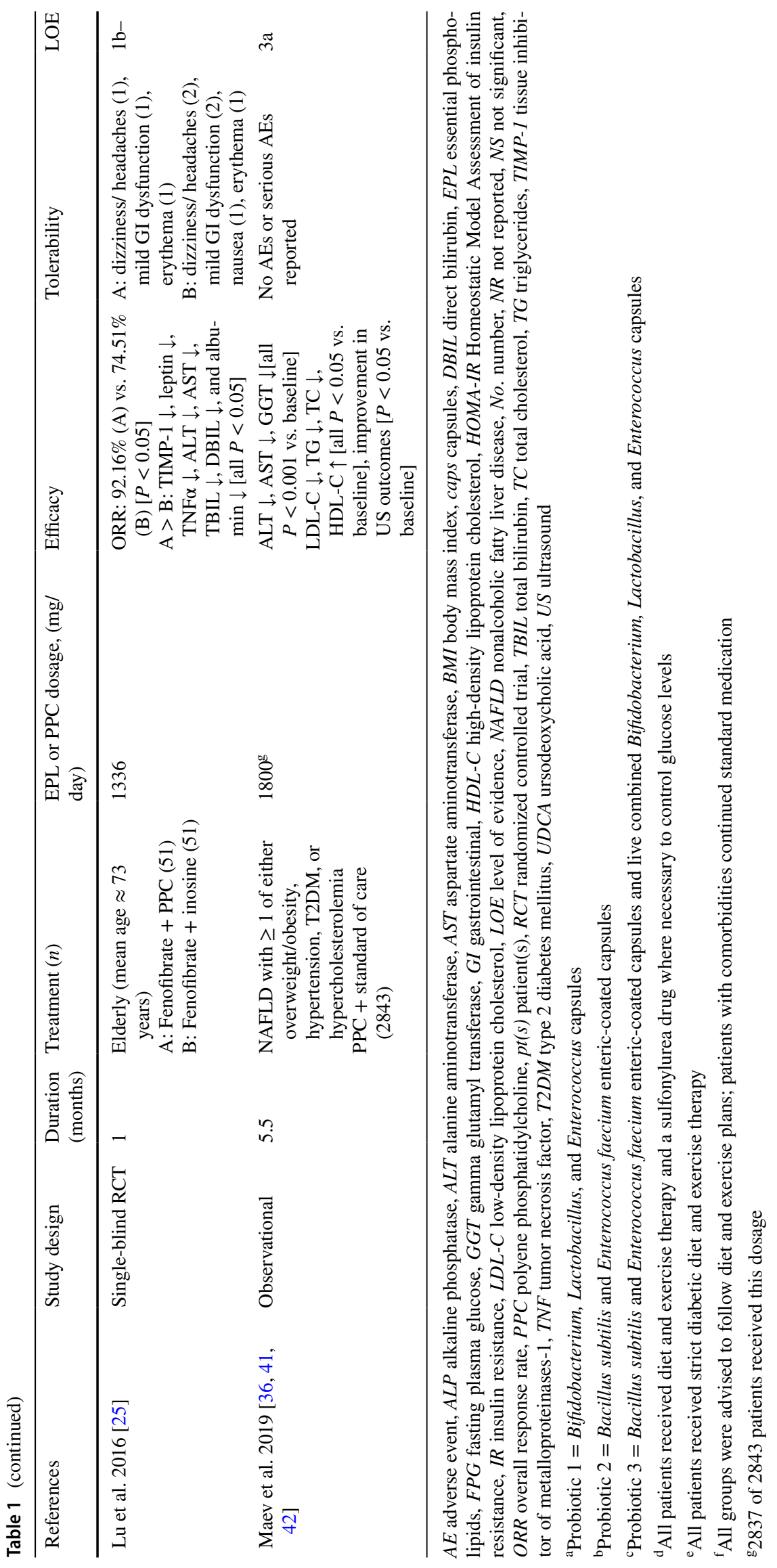


Table 2 Overview of clinical studies of oral essential phospholipids in patients with nonalcoholic steatohepatitis

\begin{tabular}{|c|c|c|c|c|c|c|c|}
\hline References & Study design & $\begin{array}{l}\text { Duration } \\
\text { (months) }\end{array}$ & Treatment $(n)$ & $\begin{array}{l}\text { EPL or PPC } \\
\text { dosage, } \mathrm{mg} / \\
\text { day }\end{array}$ & Efficacy & Tolerability & LOE \\
\hline
\end{tabular}

\section{NASH only}

Buyeverov et al. 2008 Open-label 6 [33]

Zhan et al. 2013 [32] RCT

6

A: PPC + lifestyle 1336
interventions $(n=42)$
B: PPC $(n=40)$

\section{NASH with diabetes}

$$
\text { A: } \mathrm{EPL}+\text { metformin }
$$

B: Metformin (15)

B. PPC $(n=40)$
1800

(n)

RCT

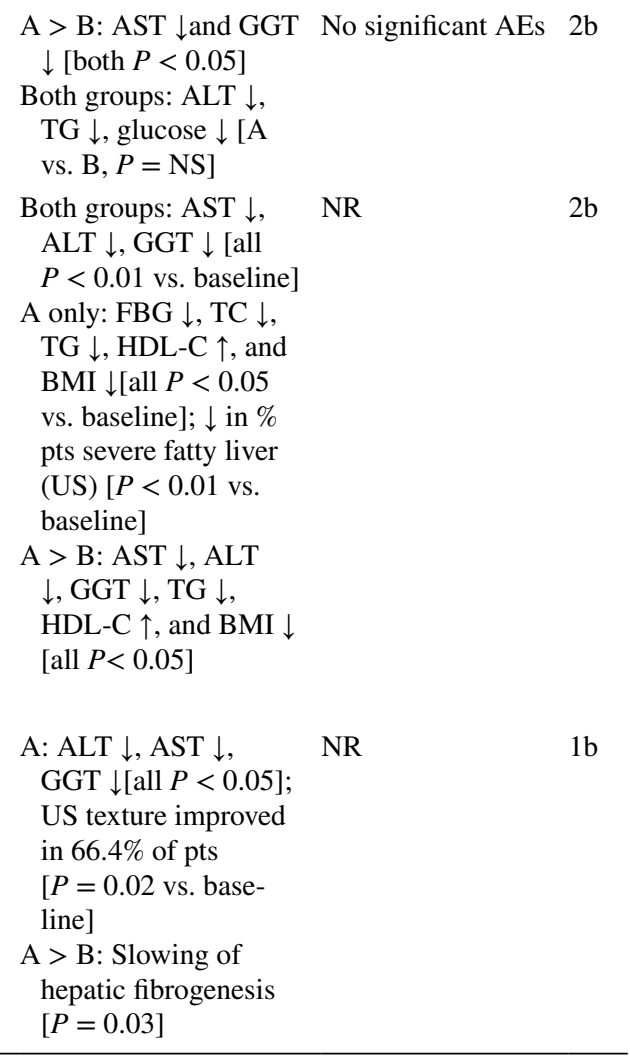

A: PPC + metformin 1368 $(152)$

B: Metformin (37)

$A E$ adverse event, $A L T$ alanine aminotransferase, $A S T$ aspartate aminotransferase, $B M I$ body mass index, $E P L$ essential phospholipids, $F B G$ fasting blood glucose, GGT gamma glutamyl transferase, $H D L-C$ high-density lipoprotein cholesterol, $L O E$ level of evidence, $N A S H$ nonalcoholic steatohepatitis, NR not reported, NS not significant, $P P C$ polyene phosphatidylcholine, pts patients, $R C T$ randomized controlled trial, TC total cholesterol, $T G$ triglycerides, $U S$ ultrasound

ultrasonography results after treatment. Additionally, elastography results showed an improvement in liver stiffness in $14.2 \%$ of patients in group A (mean reduction $3.1 \mathrm{kPa}$ [range $0.5-6$ ]), 26.1\% of patients in group B (mean -3.4 $\mathrm{kPa}$ [range $0.3-6.9$ ]), and $20.2 \%$ of patients in group $\mathrm{C}$ (mean - 3.1 kPa [range 0.5-5.5]) [37]. Improvements in liver function parameters and liver ultrasonography were also observed in the MANPOWER trial (Table 1) [41, 42]. This was despite a significant proportion of patients with diabetes mellitus and patients with overweight/obesity not receiving specific pharmacotherapy for these comorbidities (almost $15 \%$ of patients with diabetes mellitus and $40 \%$ of patients with overweight/obesity) [36].

Results of comparative studies generally confirmed that EPL given as adjunctive therapy to metformin $[23,26]$ or standard care [30] in patients with diabetes mellitus, and as an adjunctive therapy to sibutramine in patients with obesity [35], was associated with better NAFLD clinical outcomes than with comorbidity-specific treatment alone (Table 1). Effects on blood lipid levels and ultrasound results were significantly more marked in the groups receiving EPL in addition to metformin than in those receiving metformin alone [23, 26].

Results varied when EPL monotherapy was compared with other putative NAFLD treatments in patients with NAFLD [27, 29] or with NAFLD and diabetes mellitus/obesity [38]. Both UDCA [27] and metformin monotherapy [29] were significantly more effective than EPL monotherapy in terms of overall response rate and improvements in triglyceride levels and other parameters in two trials in patients with NAFLD (Table 1). In contrast, EPL was superior to UDCA for symptom reduction and liver improvements - as shown by ultrasonography —in patients with NAFLD and diabetes mellitus or obesity [38].

Both EPL monotherapy and EPL as add-on therapy to probiotics for the treatment of NAFLD provided 


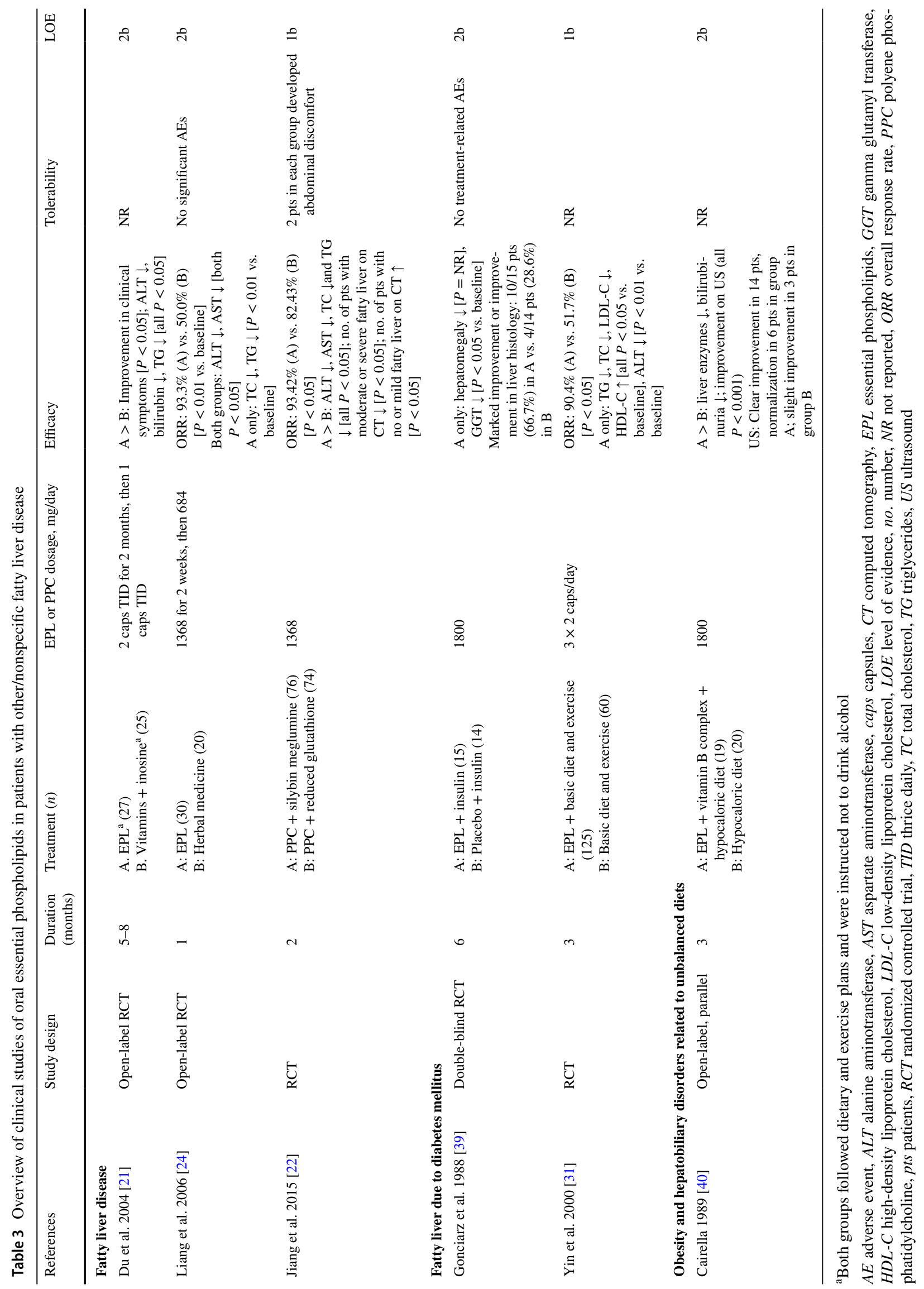


improvements from baseline in lipid levels and fatty liver (assessed by ultrasonography); however, significantly better improvements in ALT, AST, and tumor necrosis factor (TNF)- $\alpha$ levels were observed in the EPL plus probiotics group (Table 1) [28]. In the group receiving EPL monotherapy, after treatment for 30 days the proportion of patients with mild fatty liver decreased from $52 \%$ at baseline to $38 \%$, and the proportion with moderate fatty liver decreased from 48 to $40 \%$ [28].

Only one study specifically examined the efficacy and safety of EPL in elderly patients with NAFLD, evaluating EPL plus fenofibrate compared with fenofibrate plus inosine [25]. EPL plus fenofibrate was associated with a superior response rate (Table 1) and a lower rate of adverse drug reactions than fenofibrate plus inosine (but outcomes 5.9 vs. $11.7 \%)$.

\section{Nonalcoholic steatohepatitis (NASH)}

In two comparative trials, EPL in combination with metformin improved liver function in patients with NASH and diabetes mellitus [34] and in patients with NASH alone [33] (Table 2). In the latter trial, EPL plus metformin was superior to metformin monotherapy for improvements in AST and GGT levels [33]. EPL plus metformin showed a longterm advantage over metformin alone in the trial in patients with NASH and diabetes mellitus: the majority of EPL plus metformin recipients had significant reductions from baseline in ultrasonographic signs of fatty liver $(81.6 \%$ of 114$)$ and in blood glucose levels (86.0\%) [34]. This treatment group also had a significant reduction in steatosis, whereas the patients receiving metformin monotherapy had a significant increase in steatosis [34].

When EPL was added as supportive pharmacotherapy to lifestyle interventions for NASH, NAFLD outcomes were generally superior than with lifestyle interventions alone, including liver function, blood lipid levels, and BMI [32] (Table 2). The proportion of patients with severe fatty liver on ultrasonography decreased from 37.5 to $25.0 \%$ in those receiving EPL (not significant) and from 40.5 to $4.8 \%$ in those receiving EPL and lifestyle interventions $(\mathrm{P}<0.01$ vs. baseline and vs. EPL alone) [32].

\section{Other/Nonspecified fatty liver disease}

In a further six studies where the fatty liver disease was not classified as NAFLD or NASH, all were comparative, and all but two of them included fewer than 100 patients (Table 3). Overall, EPL treatment-whether as monotherapy or as add-on therapy - was associated with improvements in clinical symptoms, liver enzyme and blood lipid levels, and ultrasonography measures.
In patients with fatty liver disease, EPL was superior to vitamins plus inosine [21] and to herbal medicines [24] on some but not all liver function and serum lipid level measures (Table 1). When EPL was given as add-on therapy to other NAFLD treatments, in combination with silybin meglumine it was superior to being given in combination with reduced glutathione for most efficacy endpoints (Table 1), including liver ultrasonography assessments [22]. The number of patients with severe fatty liver on CT was reduced from 17 to 4 in group $A$ and from 16 to 0 in group B, whereas the number with moderate fatty liver decreased from 36 to 4 in group A and from 34 to 14 in group B (all $P<0.05$ vs. baseline and A vs. B). The number of patients with no fatty liver or mild fatty liver increased from 0 to 25 and from 24 to 47, respectively, in group $A$ and from 0 to 14 and from 23 to 42 , respectively, in group B (all $P<0.05$ vs. baseline and A vs. B).

In patients with fatty liver due to diabetes mellitus, EPL as add-on therapy to insulin or lifestyle intervention improved NAFLD outcomes better than insulin [39] or lifestyle intervention [31] alone. Similarly, EPL plus a hypocaloric diet was significantly more effective than a hypocaloric diet alone at improving ultrasonography, biological variables, liver enzymes, and bilirubinuria in patients with obesity and hepatobiliary disorders related to unbalanced diets [40] (Table 3).

\section{Safety}

Of the 25 studies, nine included at least some mention of safety (Tables 1, 2 and 3) [22, 24, 25, 29, 30, 33, 36, 38, 39]. However, only three studies reported any safety data [22, 25, 30]; in the other papers, the reporting was limited to a statement that there were no adverse events [36], or no adverse events that were significant [24, 29, 33], disconcerting [38], or treatment related [39].

Of the studies that reported adverse events, the most commonly reported were mild gastrointestinal events. For instance, in an 8-week, randomized, parallel-group study in patients with fatty liver disease, abdominal discomfort was reported by 2/76 patients receiving EPL plus silybin meglumine and by $3 / 74$ patients receiving EPL plus reduced glutathione; these events resolved with symptomatic treatment [22]. Moreover, where reported, the most common adverse drug reactions of EPL in elderly patients with NAFLD [25] or those with NAFLD and type 2 diabetes mellitus [30] included mild gastrointestinal dysfunction, diarrhea, or dizziness/headaches.

\section{Other supportive treatments for patients with NAFLD or NASH}

A number of other agents have been trialed in patients with NAFLD or NASH, with or without comorbidities (Fig. 1). Pharmacologic treatments for patients with NAFLD/NASH 
are often aimed at treating comorbidities, including diabetes mellitus, hypertension, obesity, and dyslipidemia [5, 12].

A summary of the efficacy/effectiveness and safety of various therapeutic approaches in NAFLD is presented in Fig. 2.

\section{Antidiabetic treatments}

NAFLD is often associated with type 2 diabetes mellitus, and a number of antidiabetic drugs have been assessed for the treatment of NAFLD. Pioglitazone has been assessed in NAFLD with or without coexisting diabetes (the latter being off label) [43] and has been shown to induce histologic improvement, including reductions in steatosis, ballooning, inflammation, and advanced fibrosis [5, 8]. However, these protective effects appear to be temporary, and relapse is common after the medicine is discontinued [44, 45]. The adverse effects of pioglitazone, namely weight gain, fracture risk, possible congestive heart failure, and bladder cancer have limited its use [43]. A number of guidelines have suggested that pioglitazone may be used in certain patients with NASH [14, 45], NASH and prediabetes or diabetes mellitus $[12,17,43]$, or NASH with advanced fibrosis [46], after careful assessment of comorbidities that may increase the risk of common adverse events. Metformin has also been shown to temporarily improve serum aminotransferases [44] but has no effect on liver fat or liver histology in patients with NAFLD [8, 12]; therefore, metformin may be used in patients with NAFLD and insulin resistance or type 2 diabetes mellitus [12] but not specifically for the treatment of NAFLD or NASH [2, 43]. Other antidiabetic drugs that may have beneficial effects in NAFLD include glucagon-like peptide-1 (GLP-1) agonists and sodium-glucose cotransporter-2 (SGLT-2) inhibitors; however, the results of further trials are needed before any recommendations can be made about their use in patients with NAFLD or NASH [5].

\section{Dietary supplements}

A number of supplements or over-the-counter preparations have been assessed in patients with NAFLD. One such agent is vitamin $\mathrm{E}$, which may be able to improve steatosis, inflammation, and hepatic ballooning in patients with NASH [45]. The potential use of vitamin $\mathrm{E}$ in patients with NASH was referred to in many guidelines, but most state that further trials are required before any firm recommendations can be made $[12,14,17,43,45]$. The long-term safety of vitamin E may be of concern [5, 14, 17, 45].

Silymarin, an antioxidant extracted from the milk thistle plant, has also shown hepatoprotective properties in patients with NAFLD [47] through its anti-inflammatory, anti-apoptotic, antifibrotic, and endocrine-metabolic and choleretic effects [47]. In a meta-analysis of small trials in patients with NAFLD (published in 2017), silymarin (as monotherapy or in combination with other agents) slightly reduced serum ALT and AST levels [48]. However, the authors concluded that these reductions were not clinically relevant and that larger, well-designed trials were necessary [48]. An earlier review (published in 2016) included five small trials in patients with NAFLD/NASH; three of these were double-blind, placebo-controlled trials (all of which were included in the above-mentioned review) and two compared silymarin with active comparators [49]. In two of the placebo-controlled trials, silymarin reduced ALT and AST levels by significantly more than placebo. In one of the active comparator trials, silymarin, pioglitazone, and metformin all significantly reduced ALT and AST levels from baseline, but pioglitazone was significantly more effective than silymarin at improving triglyceride, fasting blood sugar, and insulin levels. In the other active comparator trial, vitamin E and silymarin both significantly reduced ALT and AST levels from baseline [49]. In a more recent randomized, double-blind trial, the proportion of patients with an improvement from baseline in the NAFLD activity score did not differ significantly between silymarin and placebo [50]. The utility of orally administered silymarin is limited by the fact that absorption from the gastrointestinal tract is low (20-50\%), so the bioavailability is poor [51]. Silymarin inhibits some cytochrome P450 enzymes, meaning drug-drug interactions are possible, although they are unlikely at standard silymarin doses [51]. Silymarin is among the agents mentioned by the Chinese and Latvian NAFLD guidelines, with both noting that it is safe but that further data are required $[12,14]$. The Polish guidelines include silymarin on the list of antioxidant agents but state that, for most of these agents, there is insufficient evidence to recommend their use [17].

S-adenosyl-L-methionine (SAMe) is available as an oral dietary supplement in the USA, Canada, and Italy and as a prescription medication in several countries, including Russia, China, Germany, and India [52]. Currently, it is not commonly used for NASH or NAFLD but rather for more severe liver disease. It plays an important role in the biosynthesis of phospholipids but has poor bioavailability after oral administration [52]. A meta-analysis of 11 studies in a total of 705 patients with chronic liver disease (seven different types, and one study only in patients with NASH) showed that SAMe improved AST and total bilirubin levels but not ALT levels [53]. An observational study in 250 Indian patients showed that SAMe significantly improved signs, symptoms, and disease burden parameters associated with intrahepatic cholestasis due to NAFLD (an uncommon clinical consequence of NAFLD) [54, 55].

Other medications that may be useful in NAFLD include glutathione and glycyrrhizic acid. Oral glutathione has 


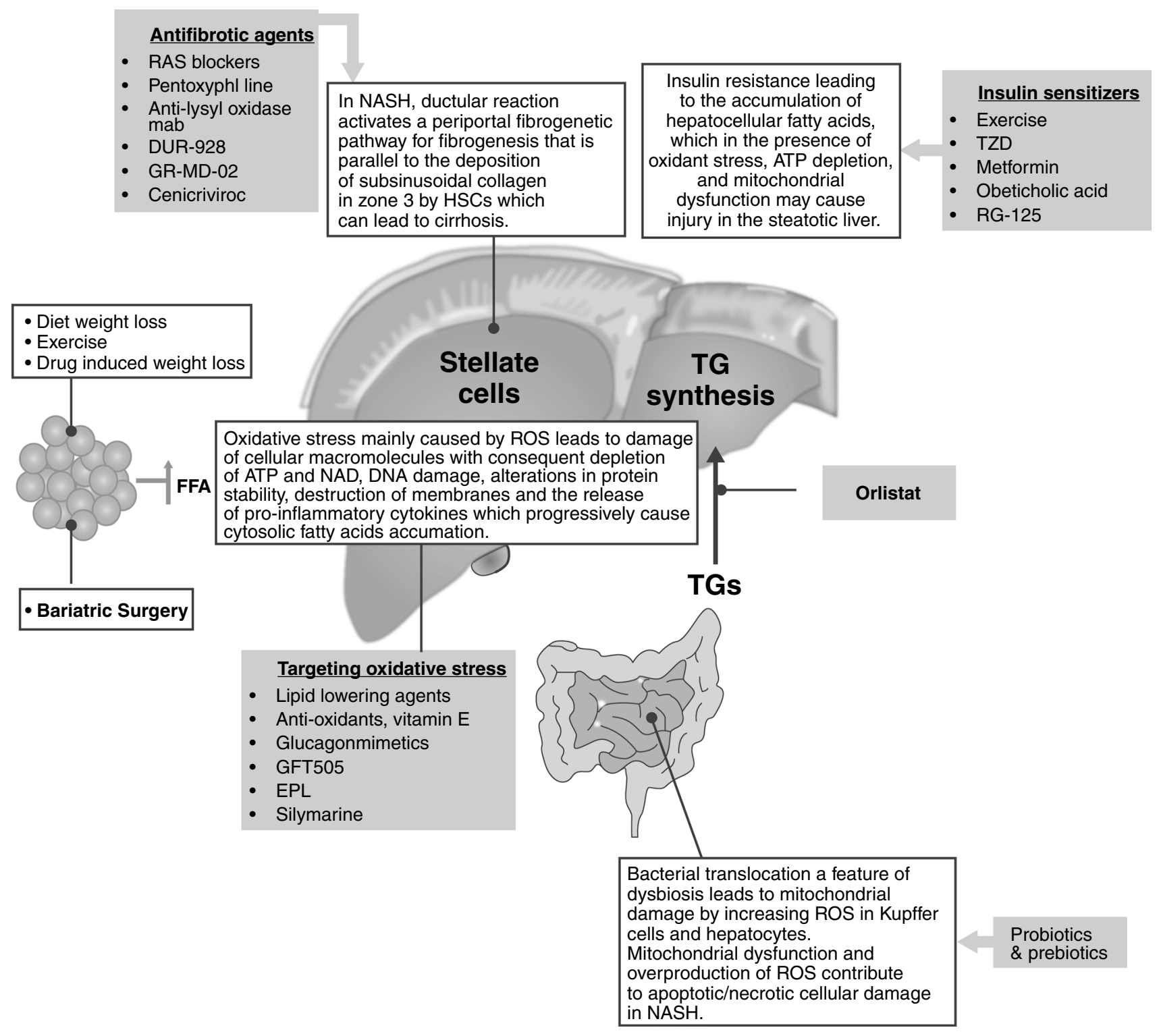

Fig. 1 Pathophysiologic mechanisms of different treatments for nonalcoholic fatty liver disease [44]. ATP adenosine triphosphate, DNA deoxyribonucleic acid, $E P L$ essential phospholipids, $F F A$ free fatty acid, HSCs hepatic stellate cells, NAD nicotinamide adenine dinu-

shown some efficacy in small pilot studies in patients with NAFLD [56, 57] or NASH [56]; however, further larger trials are needed before this treatment can be recommended. Glycyrrhizic acid (extracted from the root of the licorice plant) is commonly used in China in patients with chronic liver diseases [12], generally in combination with EPL. Studies in mice suggested that glycyrrhizic acid may be protective against NAFLD [58], and one small study showed that, as an active ingredient of licorice root, it can reduce ALT and AST levels in patients with NALFD [59]. The drug has been shown to cause edema, hypokalemia, and thrombocytopenia [59]. cleotide, $N A S H$ nonalcoholic steatohepatitis, $R A S$ renin-angiotensinsystem, ROS reactive oxygen species, $T G$ triglycerides, $T Z D$ thiazolidinediones. Reproduced from Dajani et al. [44] under the CCBY-NC agreement

\section{Bile acids}

Clinical properties of the bile acid UDCA include antiapoptotic effects, improving hepatic insulin sensitivity, and reducing serum $\mathrm{TNF} \alpha$ levels and endoplasmic reticulum stress, suggesting it may be effective in treating NAFLD. However, results of trials in patients with NAFLD or NASH have been inconsistent, and there are also toxicity concerns with UDCA, including possible genotoxicity [44]. Liver function needs to be monitored monthly during the first 3 months of UDCA therapy, followed by every 3 months thereafter [60]. 


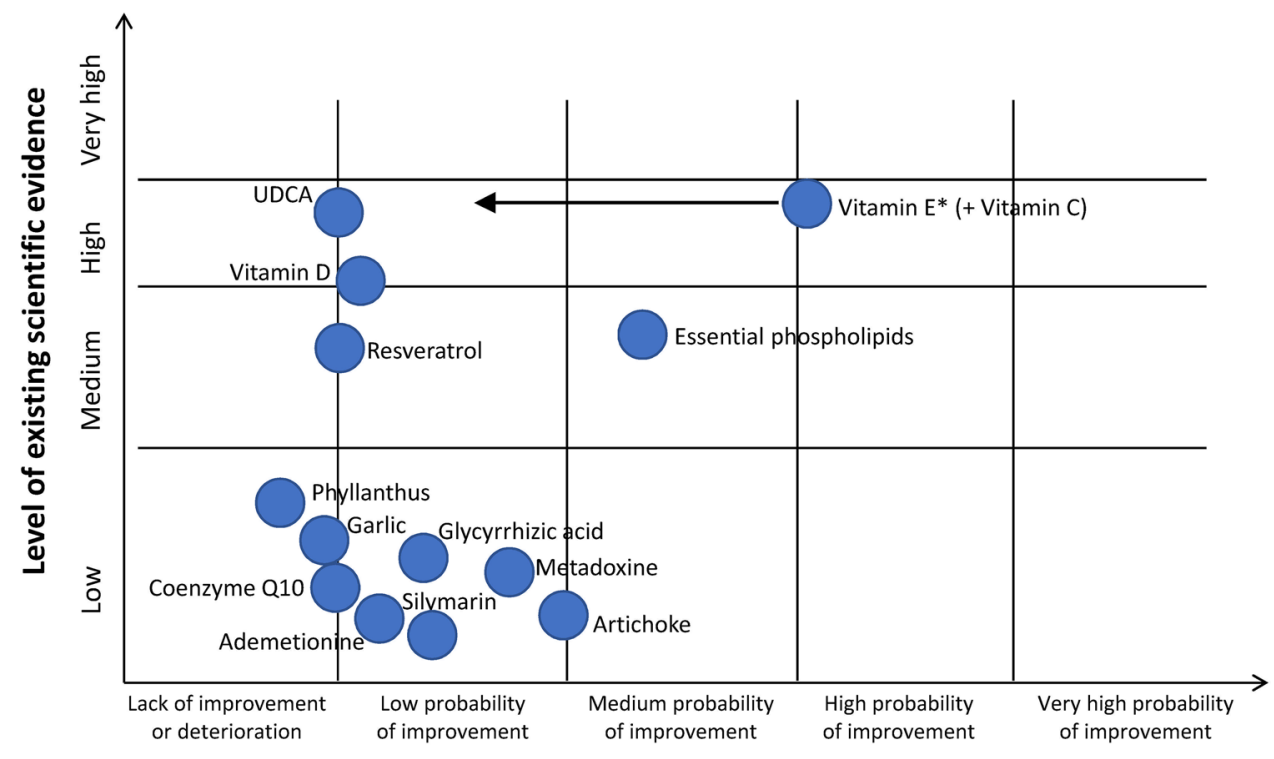

Likelihood of improvement of clinically meaningful parameters

Fig. 2 Schematic representation of the level of existing data supporting the efficacy/effectiveness and safety of various therapeutic approaches [67]. For ursodeoxycholic acid (UDCA), vitamin D, resveratrol, Phyllanthus, garlic, coenzyme Q10 (ubiquinone), ademetionine, milk thistle seed extract (Silymarin), and glycyrrhizin there are no conclusive data on the impact of these ingredients on the course of nonalcoholic fatty liver disease or there is a lack of significant difference between the effect of these ingredients and placebo/no-treatment regimens. For metadoxine and artichoke there are some limited data on therapeutic effect, but the probability of a positive impact is

\section{Investigational agents}

Phase II and III trials in patients with NAFLD are being conducted for a number of other agents, including elafibranor (a peroxisome proliferator-activated receptor [PPAR]- $\alpha$ and PPAR $\delta$ agonist), selonsertib (which inhibits apoptosis signal-regulating kinase 1 ), cenicriviroc (a $\mathrm{C}-\mathrm{C}$ motif chemokine receptor-2/5 antagonist) [5], and obeticholic acid (modified bile acid) [44].

\section{Discussion}

As the most common chronic liver disease in developed countries, with a prevalence that is increasing, NAFLD presents a significant healthcare burden $[1,3,6]$. However, although there are some promising agents in the pipeline, no pharmacologic treatment is currently specifically licensed for the treatment of NAFLD [5].

Treatment guidelines recognize the lack of specific pharmacologic agents and generally focus on lifestyle modification and treating comorbidities [12, 14, 17, 43, 45, 46]. Noting that it is off label for patients without diabetes, pioglitazone is suggested by a number of guidelines as a possible low. For vitamin $\mathrm{E}$, or vitamin $\mathrm{E}$ in combination with vitamin $\mathrm{C}$, and essential phospholipids, these active ingredients have demonstrated a positive effect on the course of nonalcoholic fatty liver disease. Evidence was considered to be at least one randomized controlled trial (RCT) with a placebo-controlled arm or no-treatment arm, and $N=50$ in the group; or at least two RCTs with a placebo or no-treatment arm as control. *However, for vitamin E, there is a potential risk of adverse effects (arrow) after long-term use at high doses. The picture is (generally provided and) adapted from Pavlov et al. [67]

treatment for patients with NASH or advanced fibrosis [12, $14,17,43,45,46]$. Many guidelines also recognize the potential of vitamin E treatment in patients with NASH [12, $14,17,43,45]$. However, there are safety concerns with both pioglitazone and vitamin E. Russian, Chinese, and Latvian guidelines suggested using an agent to limit hepatic damage, with EPL specifically recommended by the Russian and Latvian guidelines; silymarin is also recommended in the Latvian guidelines $[12,14,16]$.

EPL has been extensively evaluated in patients with NALFD. Although many of the trials covered in our review are relatively small, together they provide a body of evidence suggesting that EPL is a useful agent for the supportive treatment of patients with NAFLD. In almost all trials, EPL as monotherapy or in conjunction with other treatment improved serum levels of liver function markers and lipids and, where assessed, improved or resolved fatty liver as assessed by ultrasonography (Tables 1,2 and 3). In the largest trial reviewed here reporting on NAFLD symptoms, EPL significantly improved NAFLD symptoms, as well as reducing ALT and AST levels in patients with NAFLD only, NAFLD with diabetes mellitus, or NAFLD with hyperlipidemia [37]. In the largest trial overall, both liver enzyme levels and liver ultrasonographic features were significantly 
improved by EPL as adjunctive therapy in patients with NAFLD and comorbidities [41, 42]. In trials comparing EPL with other treatments, EPL was significantly more effective than herbal medicine [24], vitamins plus inosine [21], and sibutramine [35] and significantly more effective than UDCA in one small double-blind trial [38], but less effective in another small trial in which no blinding was reported [27]. EPL was also less effective than metformin in a small trial in patients with NAFLD, although there were improvements from baseline in both treatment arms [29].

The addition of EPL to metformin significantly improved the efficacy compared with metformin alone in two trials in patients with NAFLD and diabetes mellitus $[23,26]$ and in trials in patients with NASH [33] and NASH with diabetes mellitus [34]. The addition of EPL also significantly improved the efficacy of standard treatment in patients with NAFLD and diabetes mellitus [30], of lifestyle interventions [31] or insulin [39] in patients with fatty liver due to diabetes, and of a hypocaloric diet in patients with obesity and hepatobiliary disorders related to unbalanced diets [40]. Compared with EPL monotherapy, the efficacy of EPL was improved by the addition of probiotics in one trial [28] and of lifestyle interventions [32] in another trial. Results from a large observational study in Russia supported the premise that EPL is beneficial when used as an adjunctive therapy in patients with newly diagnosed NAFLD and metabolic comorbidities [36, 41, 42].

When safety data were reported, EPL was generally very well-tolerated with a low incidence of adverse events and no reported serious adverse events. The most commonly reported adverse events were mild gastrointestinal events.

As this is a narrative review, we did not perform any meta-analyses. However, a meta-analysis of seven trials in patients with NAFLD and obesity or type 2 diabetes mellitus has been conducted [61]. Direct meta-analysis of four studies (total $n=445$ ) showed that EPL plus antidiabetic treatment reduced levels of ALT, triglycerides, and cholesterol from baseline by significantly more than antidiabetic treatment alone. Patients were also more likely to experience overall improvement with EPL plus antidiabetic treatment than with antidiabetic treatment alone. Cohort meta-analysis of four studies (total $n=357$ ) showed that $58 \%$ of patients receiving EPL demonstrated significant clinical benefit, whereas cohort meta-analysis of two studies showed EPL reduced AST and ALT levels from baseline [61]. Additionally, a Cochrane analysis of trials of EPL in patients with NAFLD has been planned and will include studies in all languages and from any year [13].

Our review is not without limitations. First, many of the studies with EPL were small and of short duration, which prevents definitive conclusions from being drawn, particularly about the long-term efficacy of EPL. The studies were also heterogeneous with regard to design, endpoints, and concomitant therapies. Although most studies noted that patients were given dietary guidance/advice and advised to undertake exercise, the diet and exercise recommendations were not standardized between studies. Endpoints varied between studies; few used histological analysis and none used "hard" clinical endpoints such as progression to cirrhosis or cardiovascular events, so the impact of EPL on these disease outcomes has not been proven. Current surrogate endpoints such as ultrasonography and liver enzyme levels lack sensitivity, and most studies in this review used a combination of at least two surrogate endpoints to assess efficacy as recommended by regulatory authorities [62]. The 2019 recommendations on clinical study endpoints by the Liver Forum is an important step in improving clinical research standards in this field [62].

Although we conducted a comprehensive search of databases and identified studies from sources outside of these databases, we cannot be sure that further research has been undertaken that we were unable to identify. Similarly, negative studies are less likely to be published, so our data are likely to reflect this publication bias in favor of positive research, particularly in the years prior to the requirement for clinical trial registration $[63,64]$.

In this review, we have discussed biomarkers and liver function, and it should be noted that it has yet to be shown conclusively that treatments that effect these biomarkers have a resulting effect on fibrosis and mortality. While the biomarkers ALT and GGT have traditionally been viewed as being surrogate markers of liver fat [65] and may not be direct markers of histological improvement, emerging information suggests that liver fibrosis may be a dynamic process and that the rate of development of fibrosis disease is directly related to changes in disease activity [66].

In conclusion, although many of the trials were relatively small, there is a large body of evidence suggesting that EPL is a promising treatment for patients with NAFLD and is generally well-tolerated. Further large, well-designed trials on the role of EPL would be beneficial.

\section{Declarations}

Funding Medical writing support (provided by Springer Healthcare Communications following Good Publication Practice [GPP3] guidelines) and the open access fee for publication of this article was funded by Sanofi Aventis.

Conflicts of interest Asad Izziddin Dajani and Adnan Abuhammour have no conflicts of interest that are directly relevant to the content of this article.

Ethics approval This is a narrative review of published literature and the authors had no access to patients' confidential information. Therefore, no informed consent or ethical approval was required.

Author contributions Asad Izziddin Dajani contributed to the focus and scope of this review, performed the literature review, was involved 
in data analysis, reviewed the text for intellectual content, read and approved drafts, and takes responsibility for the content of the review. Adnan Abuhammour was involved in searching for and selection of relevant literature and references, was involved in data analysis, reviewed the text for intellectual content, read and approved drafts, and takes responsibility for the content of the review.

Availability of data and material Not applicable.

Code availability Not applicable.

Consent to participate Not applicable.

Consent for publication Not applicable.

Acknowledgments The authors thank Toni Dando and Tracy Harrison, both of Springer Healthcare Communications, who wrote the outline and first draft of this article, and provided editorial assistance postsubmission, respectively. This medical writing assistance, which was conducted according to Good Publication Practice (GPP3) guidelines, was provided by Springer Healthcare Communications and funded by Sanofi Aventis.

Open Access This article is licensed under a Creative Commons Attribution-NonCommercial 4.0 International License, which permits any non-commercial use, sharing, adaptation, distribution and reproduction in any medium or format, as long as you give appropriate credit to the original author(s) and the source, provide a link to the Creative Commons licence, and indicate if changes were made. The images or other third party material in this article are included in the article's Creative Commons licence, unless indicated otherwise in a credit line to the material. If material is not included in the article's Creative Commons licence and your intended use is not permitted by statutory regulation or exceeds the permitted use, you will need to obtain permission directly from the copyright holder. To view a copy of this licence, visit http://creativecommons.org/licenses/by-nc/4.0/.

\section{References}

1. Lindenmeyer CC, McCullough AJ. The natural history of nonalcoholic fatty liver disease-an evolving view. Clin Liver Dis. 2018;22(1):11-21.

2. Chalasani N, Younossi Z, Lavine JE, et al. The diagnosis and management of nonalcoholic fatty liver disease: practice guidance from the American Association for the Study of Liver Diseases. Hepatology. 2018;67(1):328-57.

3. Iqbal U, Perumpail BJ, Akhtar D, et al. The epidemiology, risk profiling and diagnostic challenges of nonalcoholic fatty liver disease. Med (Basel). 2019;6(1):41.

4. Bellentani S. The epidemiology of non-alcoholic fatty liver disease. Liver Int. 2017;37(Suppl 1):81-4.

5. Jennison E, Patel J, Scorletti E, et al. Diagnosis and management of non-alcoholic fatty liver disease. Postgrad Med J. 2019;95(1124):314-22.

6. Estes C, Razavi H, Loomba R, et al. Modeling the epidemic of nonalcoholic fatty liver disease demonstrates an exponential increase in burden of disease. Hepatology. 2018;67(1):123-33.

7. Rinella ME, Sanyal AJ. Management of NAFLD: a stage-based approach. Nat Rev Gastroenterol Hepatol. 2016;13(4):196-205.

8. Tana C, Ballestri S, Ricci F, et al. Cardiovascular risk in nonalcoholic fatty liver disease: mechanisms and therapeutic implications. Int J Environ Res Public Health. 2019;16(17):3104.
9. Rosato V, Masarone M, Dallio M, et al. NAFLD and extrahepatic comorbidities: current evidence on a multi-organ metabolic syndrome. Int J Environ Res Public Health. 2019;16(18):3415.

10. Bang KB, Cho YK. Comorbidities and metabolic derangement of NAFLD. J Lifestyle Med. 2015;5(1):7-13.

11. Glass LM, Hunt CM, Fuchs M, et al. Comorbidities and nonalcoholic fatty liver disease: the chicken, the egg, or both? Fed Pract. 2019;36(2):64-71.

12. Fan JG, Wei L, Zhuang H, et al. Guidelines of prevention and treatment of nonalcoholic fatty liver disease (2018, China). J Dig Dis. 2019;20(4):163-73.

13. Varganova DL, Pavlov CS, Casazza G, et al. Essential phospholipids for people with non-alcoholic fatty liver disease (protocol). Cochrane Database Syst Rev. 2019;4:CD013301.

14. Society of Digestive Diseases (Latvia). [Clinical practical guidelines for diagnostics, treatment and monitoring of non-alcoholic fatty liver disease]. 2020. https://www.globalliverforum.com/-/ media/Project/One-Sanofi-Web/Websites/Chc/Essentiale/LiverHealth-forum-HCP/Home/resources-for-your-clinical-practice/ Vadlinijas_en-GB.pdf. Accessed 07 Apr 2020.

15. Gundermann KJ, Gundermann S, Drozdzik M, et al. Essential phospholipids in fatty liver: a scientific update. Clin Exp Gastroenterol. 2016;9:105-17.

16. Russian Scientific Liver Society. Diagnosis and treatment of nonalcoholic fatty liver disease. 2015. http://www.rsls.ru/files/Guide lines-RSLS-NASH-2016-01-03.pdf. Accessed 06 Jan 2021.

17. Hartleb M, Wunsch E, Milkiewicz P, et al. Postępowanie z chorymi na niealkoholową stłuszczeniową chorobę wątroby: Zalecenia Polskiej Grupy Ekspertów NAFLD 2019. Med Prakt. 2019;10:47-74.

18. Gundermann KJ, Kuenker A, Kuntz E, et al. Activity of essential phospholipids (EPL) from soybean in liver diseases. Pharmacol Rep. 2011;63(3):643-59.

19. van der Veen JN, Kennelly JP, Wan S, et al. The critical role of phosphatidylcholine and phosphatidylethanolamine metabolism in health and disease. Biochim Biophys Acta Biomembr. 2017;1859(9 Bt B):1558-72.

20. Ciećko-Michalska I, Szczepanek M, Tobiasz-Adamczyk B, et al. Non-alcoholic fatty liver disease in Poland: how and at what stage is diagnosed, and how is treated. A survey study. Prz Gastroenterol. 2019;14(3):173-7.

21. Du Q. Treatment of 52 cases with hepatic dysfunctional fatty liver with Essentiale ${ }^{\circledR}$. Chin J Gastro Hepa. 2004;13:2.

22. Jiang J-Z. Clinical study on silybin meglumine combined with polyene phosphatidylcholine in treatment of fatty liver. Drugs Clinic. 2015;30(2):176-80.

23. Li Z. Efficacy of polyene phosphatidylcholine in combination with metformin for diabetes complicated with non-alcoholic fatty liver disease. Inner Mongol J Tradition Chin Med. 2013;31:10-1.

24. Liang H. Discussion of treatment of fatty liver using polyene phosphatidylcholine capsules. Chin Med Fact Mine. 2006;19:3.

25. Lu X-Y, Ye G-L. Clinical trial of polyene phosphatidylcholine in the treatment of moderate and sever fatty liver in the elderly. Chin J Clin Pharmacol. 2016;32(15):1370-5.

26. Sun C, Zheng X, Tan X, et al. Clinical observation on polyene phosphatidyl choline and metformin in the treatment of type 2 diabetes and non-alocholic fatty liver disease. Clin Focus. 2008;23(17):1272-3.

27. Wan L, Xue B. Efficacy study of ursodeoxycholic acid in the treatment of non-alcoholic fatty liver. Med Inf. 2013;26(11):605-6.

28. Wang W, Shi L, Shi L, et al. Efficacy of probiotics on the treatment of non-alcoholic fatty liver disease. Chin J Intern Med. 2018;57(2):101-7. 
29. Wu C-Y. Comparison of metformin and polyene phosphatidylcholine for nonalcoholic fatty liver disease. Pract Clin Med. 2015;16(5):3-5.

30. Wu Y. Efficacy analysis of polyene phosphatidylcholine for type 2 diabetes complicated with fatty liver. Hunan Zhong Yiyao Daxue Xuebao. 2009;29(12):41-2.

31. Yin D, Kong L. Observation for curative effect of Essentiale in treatment of fatty liver caused by diabetes mellitus. Med J Q. 2000;15:277-8.

32. Zhan G, Tan H, Li F, et al. Clinical observation of polyene phosphatidylcholine combined with lifestyle intervention in treatment of patients with non-alcoholic steatohepatitis. J Clin Hepatol. 2013;16(6):505-8.

33. Buyeverov AO, Eshanu VS, Mayevskaya MV, et al. Essential phospholipids in the complex treatment of steatohepatitis of mixed etiology. Clin Prospect Gastroenterol Hepatol. 2008;1:17-22.

34. Sas E, Grinevich V, Efimov O, et al. Benefical influence of polyunsaturated phosphatidylcholine enhances functional liver condition and liver structure in patients with nonalcoholic steatohepatitis. Results of a prolonged randomized blinded prospective clinical study. J Hepatol. 2013;58(Suppl 1):S549.

35. Sas E, Grinevich V, Kravchuk U. PTU-080 Polyunsaturated phosphatidylcholine and sibutramine decrease the liver fibrosis progress in patients with non-alcoholic liver disease. Gut. 2012;61:A216-7.

36. Maev IV, Samsonov AA, Palgova LK, et al. Real-world comorbidities and treatment patterns among patients with non-alcoholic fatty liver disease receiving phosphatidylcholine as adjunctive therapy in Russia. BMJ Open Gastroenterol. 2019;6(1):e000307.

37. Dajani AI, Abu Hammour AM, Zakaria MA, et al. Essential phospholipids as a supportive adjunct in the management of patients with NAFLD. Arab J Gastroenterol. 2015;16(3-4):99-104.

38. Arvind N, Savaikar P, Rajkumar JS. Therapy for NAFLD—a comparative study of essential phospholipids vs ursodeoxycholic acid. Indian J Clin Prac. 2006;16(10):21-4.

39. Gonciarz Z, Besser P, Lelek K, et al. Randomised placebo-controlled double-blind trial on "essential" phospholipids in the treatment of fatty liver associated with diabetes. Med Chir Digest. 1988; 17:61-85.

40. Cairella M, Callisto F, Godi R, et al. Polyunsaturated phosphatidylcholine combined with vitamin B complex in the treatment of patients with disorders of the hepatobiliary function caused by unbalanced nutrition. Clin Ter. 1989;131(4):237-46.

41. Maev IV, Samsonov AA, Palgova LK, et al. Effectiveness of phosphatidylcholine as adjunctive therapy in improving liver function tests in patients with non-alcoholic fatty liver disease and metabolic comorbidities: real-life observational study from Russia. BMJ Open Gastroenterol. 2020;7(1):e000368.

42. Maev IV, Samsonov AA, Palgova LK, et al. Effectiveness of phosphatidylcholine in alleviating steatosis in patients with non-alcoholic fatty liver disease and cardiometabolic comorbidities (MANPOWER study). BMJ Open Gastroenterol. 2020;7(1):e000341.

43. Chitturi S, Wong VW, Chan WK, et al. The Asia-Pacific working party on non-alcoholic fatty liver disease guidelines 2017part 2: management and special groups. J Gastroenterol Hepatol. 2018;33(1):86-98.

44. Dajani A, AbuHammour A. Treatment of nonalcoholic fatty liver disease: where do we stand? An overview. Saudi J Gastroenterol. 2016;22(2):91-105.

45. European Association for the Study of the Liver (EASL), European Association for the Study of Diabetes (EASD), European Association for the Study of Obesity (EASO). EASL-EASDEASO Clinical Practice Guidelines for the management of nonalcoholic fatty liver disease. J Hepatol. 2016;64(6):1388-402.
46. National Institute for Health and Care Excellence (NICE). Nonalcoholic fatty liver disease (NAFLD): assessment and management (NICE Guidelines). 2016. https://www.nice.org.uk/guida nce/ng49. Accessed 15 Nov 2019.

47. Cicero AFG, Colletti A, Bellentani S. Nutraceutical approach to non-alcoholic fatty liver disease (NAFLD): the available clinical evidence. Nutrients. 2018;10(9):1153.

48. de Avelar CR, Pereira EM, de Farias Costa PR, et al. Effect of silymarin on biochemical indicators in patients with liver disease: systematic review with meta-analysis. World J Gastroenterol. 2017;23(27):5004-17.

49. Hellerbrand C, Schattenberg J, Peterburs P, et al. The potential of silymarin for the treatment of hepatic disorders. Clinical Phytoscience. 2016;2:7.

50. Wah Kheong C, Nik Mustapha NR, Mahadeva S. A randomized trial of silymarin for the treatment of nonalcoholic steatohepatitis. Clin Gastroenterol Hepatol. 2017;15(12):1940-9.

51. Javed S, Kohli K, Ali M. Reassessing bioavailability of silymarin. Altern Med Rev. 2011;16(3):239-49.

52. Lu SC, Mato JM. S-adenosylmethionine in liver health, injury, and cancer. Physiol Rev. 2012;92(4):1515-42.

53. Guo T, Chang L, Xiao Y, et al. S-adenosyl-L-methionine for the treatment of chronic liver disease: a systematic review and metaanalysis. PLoS ONE. 2015;10(3):e0122124.

54. Virukalpattigopalratnam MP, Singh T, Ravishankar AC. Heptral (ademetionine) in patients with intrahepatic cholestasis in chronic liver disease due to non-alcoholic liver disease: results of a multicentre observational study in India. J Indian Med Assoc. 2013;111(12):856-9.

55. Choudhuri G, Singh T. Heptral ${ }^{\circledR}$ (ademetionine) in patients with chronic alcoholic liver disease: results of a multicentre observational study in Indian patients. Int J Res Health Sci. 2014;2(3):831-41.

56. Irie M, Sohda T, Anan A, et al. Reduced glutathione suppresses oxidative stress in nonalcoholic fatty liver disease. Euroasian $\mathrm{J}$ Hepatogastroenterol. 2016;6(1):13-8.

57. Honda Y, Kessoku T, Sumida Y, et al. Efficacy of glutathione for the treatment of nonalcoholic fatty liver disease: an openlabel, single-arm, multicenter, pilot study. BMC Gastroenterol. 2017;17(1):96.

58. Sun X, Duan X, Wang C, et al. Protective effects of glycyrrhizic acid against non-alcoholic fatty liver disease in mice. Eur J Pharmacol. 2017;806:75-82.

59. Rostamizadeh $P$, Mazloom $Z$. The effect of licorice and probiotics on nonalcoholic fatty liver disease (NAFLD): a systematic review. Int J Nutr Sci. 2019;4(4):163-9.

60. European Medicines Agency. Ursodeoxycholic acid. Summary of product characteristics. 2018. https://mhraproducts4853.blob. core.windows.net/docs/2b33d8ba3401cc39e0b57d5ca5bbb9f24a8 ef454. Accessed 19 Jan 2021.

61. Dajani AI, Popovic B. Essential phospholipids for nonalcoholic fatty liver disease associated with metabolic syndrome: a systematic review and network meta-analysis. World J Clin Cases. 2020;8(21):5235-49.

62. Cheung A, Neuschwander-Tetri BA, Kleiner DE, et al. Defining improvement in nonalcoholic steatohepatitis for treatment trial endpoints: recommendations from the liver forum. Hepatology. 2019;70(5):1841-55.

63. Dickersin K. The existence of publication bias and risk factors for its occurrence. JAMA. 1990;263(10):1385-9.

64. Easterbrook PJ, Berlin JA, Gopalan R, et al. Publication bias in clinical research. Lancet. 1991;337(8746):867-72.

65. Preiss D, Sattar N, Harborne L, et al. The effects of 8 months of metformin on circulating GGT and ALT levels in obese 
women with polycystic ovarian syndrome. Int $\mathrm{J}$ Clin Pract. 2008;62(9):1337-43.

66. Kleiner DE, Brunt EM, Wilson LA, et al. Association of histologic disease activity with progression of nonalcoholic fatty liver disease. JAMA Netw Open. 2019;2(10):e1912565.
67. Pavlov CS, Kuznetsova EA, Shulpekova YO, et al. Treatment principles. In: Nonalcoholic liver disease. Moscow: GEOTAR-Media; 2019. pp. 46-57. 